\title{
Imbalance Detection of Rotor Rotational Speed Using Doppler Radar
}

\author{
Young-Jae Choi $\cdot$ In-Sik Choi*
}

\begin{abstract}
In operating a wind turbine, both predictive and condition-based maintenances are required to minimize the downtime caused by maintenance. The imbalance of rotor rotational speed is an important factor for diagnosing wind turbine failures. The rotational speed imbalance can be caused by accumulated damage or the accumulation of ice, dust, and moisture. In this paper, we proposed a method for detecting the rotational speed imbalance of a wind turbine using a Doppler radar. We calculated the difference in the rotational speed for different times using spectrograms obtained by observing the wind turbine with a Doppler radar and determined the rotational speed imbalance using the fast Fourier transform. The performance of the proposed algorithm was verified using both synthetic and numerical data.
\end{abstract}

Key Words: Doppler Radar, Dynamic Radar Cross-Section, Imbalance Detection, Spectrogram, Wind Turbine.

\section{INTRODUCTION}

The wind power market has grown rapidly over the past decade. Although wind power is a well-known eco-friendly energy source, the associated operating and maintenance costs are high [1]. In particular, wind turbines are expensive to maintain because they require expensive cranes to lift the equipment [1]. In general, predictive maintenance (PdM) and condition-based maintenance $(\mathrm{CBM})$ are required to minimize the downtime of wind turbines due to maintenance [1]. To realize the PdM and CBM, a condition monitoring system is often required. For wind turbines, the main failures can be found in the gearboxes, bearings, generators, rotors, blades, and pitch control systems [1, 2]. Among these failures, rotor imbalance can be caused by damage accumulated on the rotor blades or by the accumulation of uneven ice, dust, and moisture [2]. Regarding direct-drive wind turbines, analyzing the current output of the generator can detect rotor imbalance [1]. This is because a direct-drive wind turbine can directly affect the output current of the rotor. From previous studies, power spectral density analysis, bicoherence analysis, time-frequency analysis, amplitude demodulation, and data mining have been used for the imbalance fault detection of wind turbines [3]. It is clear that data can be obtained when a sensor is installed on the rotor axis [4]; however, sensor installation remains a very difficult task [1]. It is expected that if the rotational motion of the blade can be directly observed, many shortcomings of the existing methods can be overcome. However, since the wind turbine blade is a large structure of several tens of meters, it is complex to accurately observe the rotational motion of the blade. In the past decade, radars have been used in many studies to measure wind turbines. Laboratory measurements of spectrograms for wind turbine scale models were reported

\footnotetext{
Manuscript received July 25, 2020 ; Revised October 31, 2020 ; Accepted November 16, 2020. (ID No. 20200725-109J)

Department of Electrical \& Electronic Engineering, Hannam University, Daejeon, Korea.

"Corresponding Author: In-Sik Choi (e-mail: recog@hnu.kr)
}

This is an Open-Access article distributed under the terms of the Creative Commons Attribution Non-Commercial License (http://creativecommons.org/licenses/by-nc/4.0) which permits unrestricted non-commercial use, distribution, and reproduction in any medium, provided the original work is properly cited.

(c) Copyright The Korean Institute of Electromagnetic Engineering and Science. All Rights Reserved. 
in 2010 and 2013 [5-8]. From 2015 to 2017, studies have been conducted to directly measure the industrial wind turbine to obtain the spectrogram [9-11]. Many studies have examined the performance of structural health monitoring of wind blades using noncontact sensors. Many types of noncontact sensors have been used in structural health monitoring, including infrared thermography, laser sensors, and microwaves [12]. The requirement for many of these sensors is that the target rotors are stationary or only move slowly during the measurements. In particular, the radarbased method has shown good performance. Since this method requires a wide bandwidth, the system is expensive; thus, more affordable methods require exploration. It is important to note from these studies that Doppler radar can be used to construct a system to observe the entire blade of an industrial wind turbine at very low costs [10]. Also, it was commonly reported that a unique flash component can be found in the spectrogram of a rotating wind blade [9-11]. The occurrence of the flash component is the most evident characteristic of a wind blade and is closely related to its motion state [9-11]. In this study, we propose a method for detecting the imbalance of the wind turbine blade with the spectrogram obtained using Doppler radar. We describe the phenomenon that occurs when a wind-turbine blade rotates at an unbalanced speed. Then, we explain the effect of this phenomenon on the obtained spectrogram. Also, we introduce an algorithm that can detect the imbalance using these characteristics. Finally, the proposed method is verified using both synthetic and numerical data.

\section{PROPOSED METHOD}

\section{Derivation of the Algorithm}

Wind turbine blades are normally large structures of several tens of meters and are affected by gravity during rotation. The mass balance between the three blades of a wind turbine is critical for the blades to rotate stably under gravity. Fig. 1 illustrates a situation in which the mass balance of blades is violated $[3,13]$.

In Fig. 1, it is assumed that the mass of each blade is $M$, except that one blade has an additional mass of $m$. Any slight differences between the masses of the three blades are neglected. When mass $M$ is removed from each blade, the model can be simplified to have only one blade with mass $m$ [3]. In the simplified model, the change in angular acceleration during rotation is illustrated in Fig. 2.

In Fig. 2(a), the maximum acceleration is applied when the rotating angle is $0^{\circ}$, while the maximum deceleration is applied at $180^{\circ}$. Therefore, the angular acceleration at different blade rotation angles is cosinusoidal function (Fig.

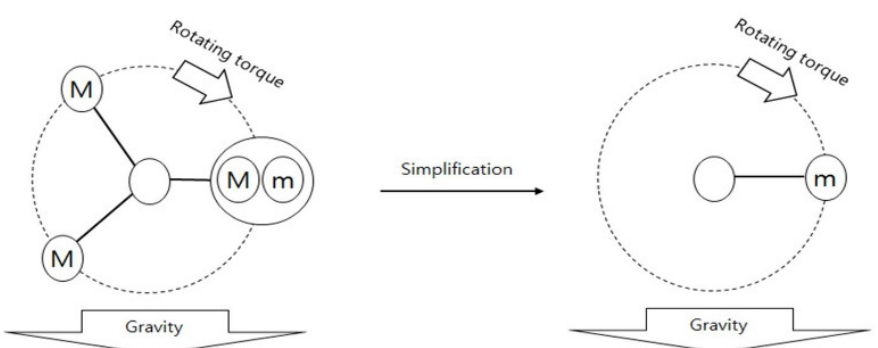

Fig. 1. Illustrative model of the wind turbine blades with unbalanced masses.

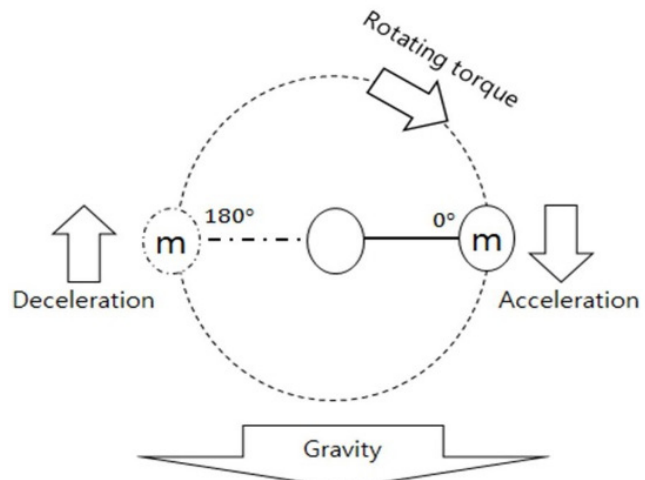

(a)

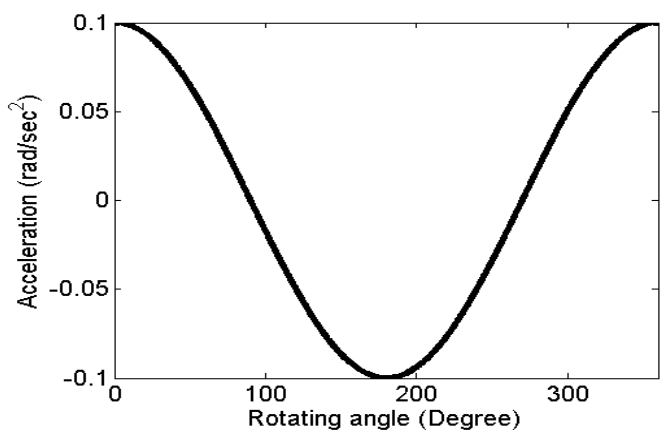

(b)

Fig. 2. Change in the acceleration of imbalanced blades: (a) the simplified model and (b) acceleration.

2(b)). Fig. 2(a) is referred to as a mass model in related studies $[3,14]$. From Eq. (9) of [14], the angular acceleration is derived below:

$$
a(t)=z \cos (2 \pi X t)
$$

where $X$ is the revolution per second (rps) of the rotor, $t$ is the time sample, and $z$ is a variable that determines the amount of change in acceleration. $z$ is proportional to the additional mass. The rotational speed of the wind blade, which is changed by the acceleration of Eq. (1), can be obtained as

$$
\Omega(t)=2 \pi X+\int_{0}^{t} z \cos (2 \pi X t) d t=2 \pi X+\frac{z \sin (2 \pi X t)}{2 \pi X}
$$


Adding the base rotational speed $2 \pi X$ to Eq. (2) and integrating it regarding $t$, we can obtain the rotation angle of the wind turbine blade regarding time as

$$
\begin{aligned}
\theta(t)=\int_{0}^{t}(2 \pi X & \left.+\frac{z \sin (2 \pi X t)}{2 \pi X}\right) d t \\
& =2 \pi X t-\frac{z \cos (2 \pi X t)}{4 \pi^{2} X^{2}}
\end{aligned}
$$

Eq. (4) can be obtained by removing the term $2 \pi X t$ from Eq. (3).

$$
\theta(t)-2 \pi X t=-\frac{z \cos (2 \pi X t)}{4 \pi^{2} X^{2}}
$$

The Fourier transform is applied to the left side of Eq. (4), and we can obtain

$$
\begin{gathered}
\Theta\left(X_{n}\right)=\left|\int_{-T / 2}^{T / 2}-\frac{z \cos \left(\Omega_{0} t\right)}{\Omega_{0}^{2}} \exp \left(-j 2 \pi X_{n} t\right) d t\right|= \\
\left|-\frac{z\left(\frac{1}{2} \sin c\left(\pi X T-\pi T X_{n}\right)-\frac{1}{2} \sin c\left(\pi X T+\pi T X_{n}\right)\right)}{4 \pi^{2} X^{2}}\right|= \\
\left|\frac{\frac{z}{2} \sin c\left(\pi X T+\pi T X_{n}\right)}{4 \pi^{2} X^{2}}\right|+\left|\frac{\frac{z}{2} \sin c\left(\pi X T-\pi T X_{n}\right)}{4 \pi^{2} X^{2}}\right|
\end{gathered}
$$

In Eq. (5), $X$ is always positive, and thus, Eq. (5) can be simplified to

$$
\Theta\left(X_{n}\right)=\left|\frac{\frac{Z}{2} \operatorname{sinc}\left(\pi X T-\pi T X_{n}\right)}{4 \pi^{2} X^{2}}\right|
$$

where $\Theta\left(X_{n}\right)$ reaches its maximum value when $X_{n}=X$, and $z$ is always positive. By substituting $X_{n}=X$ into Eq. (6), we obtain

$$
z=8 \pi^{2} X^{2} \Theta(X)
$$

\section{Parameter Estimation Algorithm}

From Eqs. (3) to (7), $z$ and $X$ in Eq. (1) can be estimated from $\theta(t)$ in Eq. (3), and $\theta(t)$ can be obtained from the spectrogram. Fig. 3 shows the relationship between the rotation angle and the spectrogram of a rotating wind blade.

In Fig. 3(b), a flash occurs in the positive Doppler direction whenever the blade passes the $0^{\circ}$ point. Regarding the three blades, the angle between each blade is $120^{\circ}$ and, therefore, the rotation angle between each flash is $120^{\circ}$.

The time sample in which the $n$-th flash occurs is substituted into $t_{n}$, and the rotation angle corresponding to the $n$-th flash is calculated using

$$
\theta\left(t_{n}\right)=\frac{2 \pi}{3} n,
$$

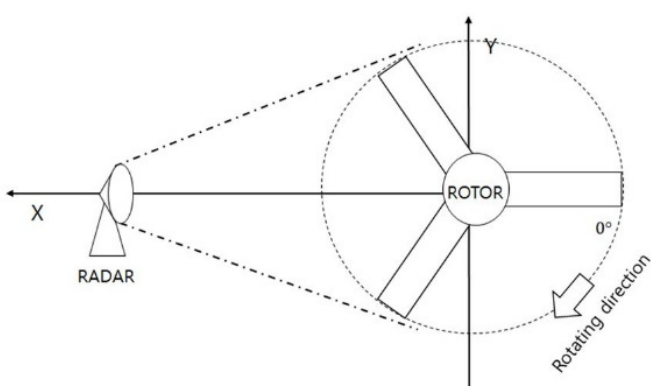

(a)

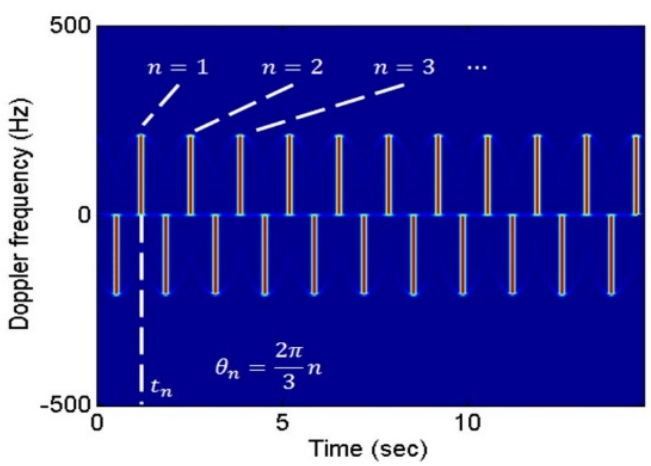

(b)

Fig. 3. Relationship between the rotation angle and the spectrogram of a rotating wind blade: (a) the geometry of the rotating rotor blade and radar and (b) the spectrogram of a rotating rotor blade.

where $n=1,2, \cdots, N$, and $N$ is the number of flashes on the positive Doppler direction of the spectrogram.

From Eq. (8), we obtain $\dot{X}$ and $b$ that satisfy the condition given by

$$
\dot{X}=\min _{X, b} \sum_{n=1}^{N}\left(\theta\left(t_{n}\right)-\left(2 \pi \dot{X} t_{n}+b\right)\right)^{2},
$$

where $b$ is the initial rotation angle, $\dot{X}$ can be obtained by the least square solution according to [15], as given by

$$
\vec{x}=\left[\begin{array}{c}
2 \pi \dot{X} \\
b
\end{array}\right]=\left(\bar{A}^{T} \bar{A}\right)^{-1} \bar{A}^{T} \vec{\theta},
$$

where $\vec{\theta}=\left[\theta\left(t_{n}\right)\right] \in \mathbb{R}^{N}$ and $\bar{A}=\left[\begin{array}{ll}t_{n} & 1_{n}\end{array}\right] \in \mathbb{R}^{N \times 2}$. For $\vec{\theta}$, the primary and direct current components obtained in Eq. (10) are removed and the frequency spectrum is obtained using

$$
\Theta\left(X_{\mathrm{j}}\right)=\left|\vec{D}_{J}^{T}(\vec{\theta}-\bar{A} \vec{x})\right|,
$$

where $\overrightarrow{D_{J}}=\left[\exp \left(-j 2 \pi X_{\mathrm{j}} t_{n}\right)\right] \in \mathbb{C}^{N}$ and $\vec{\Theta}=\left[\Theta\left(X_{\mathrm{j}}\right)\right] \epsilon$ $\mathbb{R}^{J}, J$ is the number of samples on the frequency axis, and $X_{\mathrm{j}}=1.5(j-1) \frac{\dot{X}}{J}$. From Eq. (11), the element with the maximum value is located at $\dot{X}$, and its value is equal to

$$
\Theta(\dot{X})=\max \vec{\Theta}
$$


Substituting $\Theta(\dot{X})$ into Eq. (7), and $z$ can be estimated by

$$
\dot{z}=8 \pi^{2} X^{2} \Theta(\dot{X})
$$

\section{Flash Extraction from Spectrogram}

The proposed method can accurately extract the interval between two flashes that appear in the spectrogram. The signal vector of the dynamic radar cross-section can be obtained by $\vec{s}=\left[s_{p}\right] \in \mathbb{C}^{P}$. The spectrogram $\bar{S}=\left[S_{q, p}\right] \in$ $\mathbb{R}^{Q \times P}$ can be obtained by performing a short-time Fourier transform on $\vec{s}$ [16], where $Q$ is the number of Doppler frequency samples and $P$ is the number of time samples. The spectrogram can be converted to a one-dimensional vector using

$$
U_{p}=\sum_{q=1}^{Q} S_{q, p} F_{q},
$$

where $F_{q}$ is the $q$-th sample of the Doppler frequency and $\vec{U}=\left[U_{p}\right] \in \mathbb{R}^{P}$ is the vector of one-dimensional signals obtained from Eq. (14). Fig. 4 shows the one-dimensional graph obtained by substituting the spectrogram of Fig. 3(b) into Eq. (14).

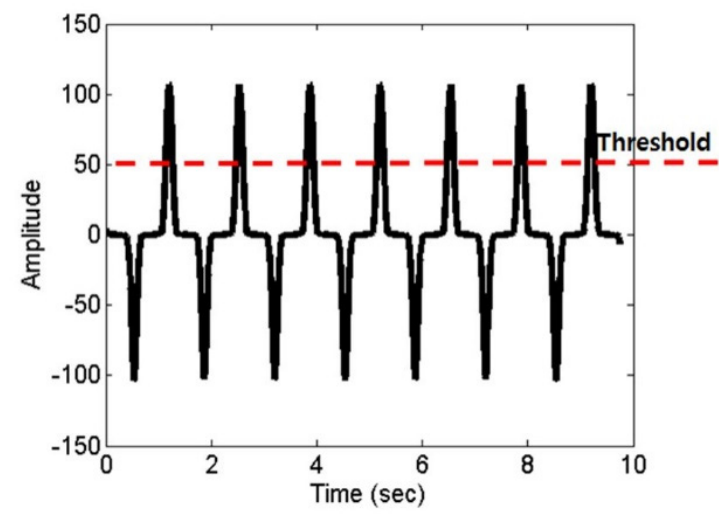

(a)

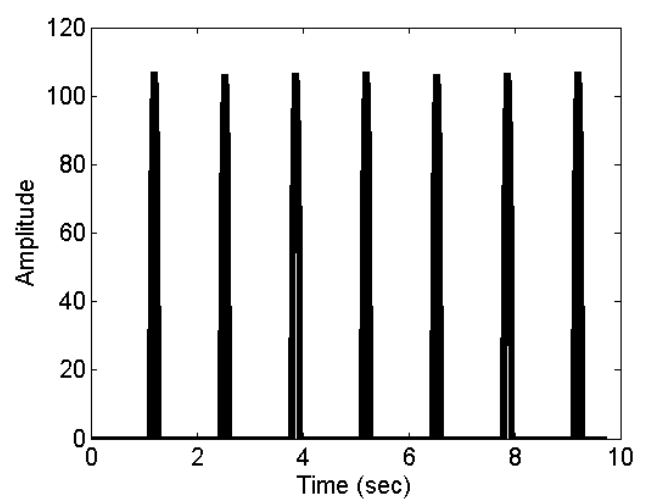

(b)

Fig. 4. One-dimensional graph: (a) example of the onedimensional graph and (b) the graph after applying threshold.
The location of the flash in the spectrogram in Fig. 3(b) is the same as the peak location in the one-dimensional graph in Fig. 4(a). A threshold is applied to remove unwanted components by

$$
U_{p}^{m}=\left\{\begin{array}{cc}
U_{p} & \text { others } \\
0 & U_{p}<B
\end{array}\right.
$$

where $p=1,2, \cdots P, m=1$ is the iteration index, and $B$ is the threshold value. In this study, we use $B=\frac{U_{\max }}{2}$ and $U_{\text {max }}=\max \vec{U}$.

The peak is removed using the following procedure:

Step 1. Defining a vector $\overrightarrow{U_{m}}=\left[U_{p}^{m}\right] \in \mathbb{R}^{P}$ using $U_{p}^{m}$, and initializing it from $m=1$;

Step 2. Using Eq. (16) to find $p$ that makes $\overrightarrow{U_{m}}$ the largest;

Step 3. Removing the extracted component from $\overrightarrow{U_{m}}$ using Eq. (17);

Step 4. Updating with $m=m+1$;

Step 5. Repeating Steps 2-4 until there is no component to extract;

Step 6. Sorting $\vec{K}=\left[K_{m}\right] \in \mathbb{R}^{P}$ in ascending order. $\vec{K}=$ $\left[K_{p}\right] \in \mathbb{R}^{P}$ is the vector of time index, where $p=$ $1,2, \cdots P$, and $p$ is the index assigned to the element while sorting $\vec{K}$. By substituting $n=K_{p}$ into Eq. (8), we obtain $t_{n}=t\left(K_{p}\right)$ and $\theta\left(t_{n}\right)$.

$$
\begin{gathered}
K_{m}=\max _{p} \overrightarrow{U_{m}} \\
U_{p}^{m+1}=\left\{\begin{array}{cc}
U_{p}^{m} & \text { others } \\
0 & p_{\text {min }}<p<p_{\text {max }}
\end{array}\right. \\
p_{\text {min }}=\left\{\begin{array}{cc}
K_{m}-2 W & , \text { others } \\
1 & \left(K_{m}-2 W\right)<1
\end{array}\right. \\
p_{\text {max }}=\left\{\begin{array}{cc}
K_{m}+2 W & , \text { others } \\
P & \left(K_{m}+2 W\right)>P
\end{array}\right.
\end{gathered}
$$

where $W$ is the number of time samples corresponding to the width of the flash in the spectrogram.

\section{SIMULATION}

\section{Synthetic Data}

Assuming that the radar observes the wind turbine from the side, the point scatter model of a rotor with three blades can be simply expressed as in [17]:

$$
\mathrm{s}_{p}=\sum_{k=0}^{2} \operatorname{sinc}\left(\Phi_{\mathrm{k}}(p)\right) \exp \left\{j \Phi_{\mathrm{k}}(p)\right\}
$$


where $\Phi_{\mathrm{k}}(p)$ is given in [17] as

$$
\Phi_{\mathrm{k}}(p)=\frac{4 \pi f}{c} \frac{L}{2} \cos \left(\Omega t_{p}+k \pi \frac{2}{3}+\frac{\pi}{2}\right),
$$

where $\Omega t_{p}$ is the rotational angle at $t_{p}$. In our problem, the rotational speed, $\Omega$, changes over time. Thus, $\theta(t)$ should be used instead of $\Omega t_{p} . \theta(t)$ is given by Eq. (3). Eq. (22) can be obtained by substituting $\theta(t)$ into Eq. (21).

$$
\begin{gathered}
\Phi_{\mathrm{k}}(p)= \\
\frac{4 \pi f}{c} \frac{L}{2} \cos \left(k \pi \frac{2}{3}+\frac{\pi}{2}+2 \pi X t_{p}-\frac{z \cos \left(2 \pi X t_{p}\right)}{4 \pi^{2} X^{2}}\right),
\end{gathered}
$$

where $L$ is the radius of the rotor, $f$ is the radar transmission frequency, and $c$ is the speed of light in the free space. Table 1 lists the parameters for the simulation in this study. Fig. 3(a) shows the geometry of the simulation.

In Table $1, f_{s}$ is a sampling frequency. Fig. 5 shows the frequency spectrum $\Theta\left(X_{j}\right)$ calculated by applying the proposed algorithm to the composite data using the parameters listed in Table 1. As illustrated in Fig. 5, the peak occurs at $0.28 \mathrm{rps}$ in the rotation period of the wind blade. As $z$ increases, higher peaks occur at the corresponding positions, and therefore, it is possible to determine the degree of wind turbine imbalance by checking the peaks. In Fig. 5, the peak value can be substituted into Eq. (7) to calculate $z$, and the

Table 1. Parameters of the synthetic simulation

\begin{tabular}{cc}
\hline Parameter & Value \\
\hline$L$ & $11 \mathrm{~m}$ \\
$f$ & $0.28 \mathrm{rps}$ \\
$f_{s}$ & $1 \mathrm{GHz}$ \\
$P$ & $1,200 \mathrm{sps}$ \\
$t_{P}$ & 144,000 \\
\hline
\end{tabular}

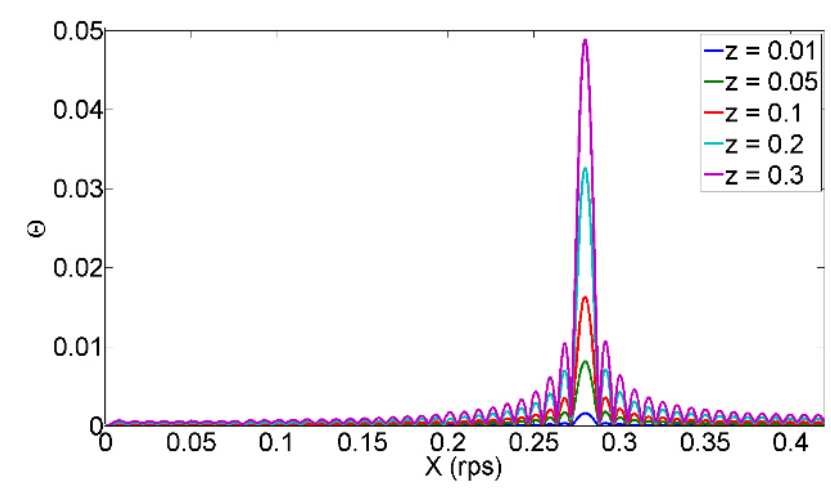

Fig. 5. Frequency spectrum for different $z\left(\mathrm{rad} / \mathrm{sec}^{2}\right)$ values $(X=$ $0.28 \mathrm{rps}$ ). results are as listed in Table 2.

It can be summarized in Table 2 that the estimated $z$ shows an error below 2\%, compared to the actual $z$. However, for $z=0.01$, where the amplitude of the acceleration is small, the error exceeds $4 \% . z=0.01$ is the limit of the $z$ value that can be extracted under the conditions of Table 1 . By increasing the sampling frequency and observation time, the precision of the observation value can be improved.

Fig. 6 illustrates the frequency spectrum when the condition is changed to $X=0.2$ rps.

As illustrated in Fig. 6, the peak occurs at $0.2 \mathrm{rps}$ in the rotation cycle of the wind blade. However, $\Theta\left(X_{j}\right)$ for different $z$ values is found to differ from that in Fig. 5. Table 3 summarizes the $z$ values calculated by substituting the peak value obtained in Fig. 6 into Eq. (7).

Table 2. Estimated parameters of the synthetic simulation $(X=$ $0.28 \mathrm{rps})$

\begin{tabular}{ccc}
\hline $\begin{array}{c}\text { True } z \\
\left(\mathrm{rad} / \mathrm{sec}^{2}\right)\end{array}$ & $\begin{array}{c}\text { Estimated } z \\
\left(\mathrm{rad} / \mathrm{sec}^{2}\right)\end{array}$ & $\begin{array}{c}\text { Relative error for } z \\
(\%)\end{array}$ \\
\hline 0.01 & 0.0096 & 4.43 \\
0.05 & 0.0504 & 0.84 \\
0.1 & 0.1007 & 0.73 \\
0.2 & 0.2022 & 1.11 \\
0.3 & 0.3046 & 1.54 \\
\hline
\end{tabular}

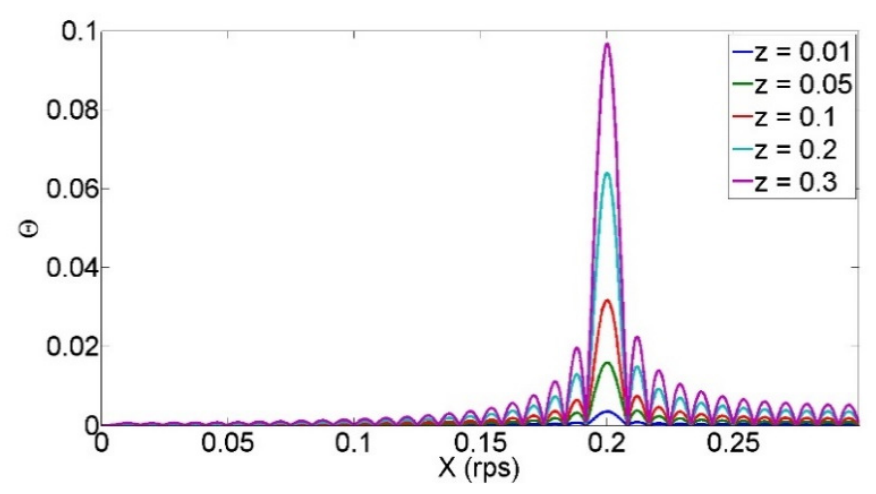

Fig. 6. Frequency spectrum for different $z\left(\mathrm{rad} / \mathrm{sec}^{2}\right)$ values $(X=$ $0.2 \mathrm{rps})$.

Table 3. Estimated parameters of the synthetic simulation $(X=$ $0.2 \mathrm{rps})$

\begin{tabular}{ccc}
\hline $\begin{array}{c}\text { True } z \\
\left(\mathrm{rad} / \mathrm{sec}^{2}\right)\end{array}$ & $\begin{array}{c}\text { Estimated } z \\
\left(\mathrm{rad} / \mathrm{sec}^{2}\right)\end{array}$ & $\begin{array}{c}\text { Relative error for } z \\
(\%)\end{array}$ \\
\hline 0.01 & 0.0110 & 10.28 \\
0.05 & 0.0507 & 1.43 \\
0.1 & 0.1005 & 0.45 \\
0.2 & 0.2039 & 1.97 \\
0.3 & 0.3054 & 1.79 \\
\hline
\end{tabular}


From Table 3 , the estimated $z$ also shows an error below $2 \%$, compared to the actual $z$. However, for $z=0.01$, where the amplitude of the acceleration is small, the error exceeds $10 \%$.

\section{Numerical Data}

The numerical data are calculated using the physical optics method by reflecting the rotational speed obtained using Eq. (2) [18]. Table 4 illustrates the parameters used for the simulation. Doppler radar used a continuous wave and a horizontal polarization. The material of wind turbine was considered a perfect electric conductor.

Fig. 7 illustrates the spectrogram of the numerical data. At signal-to-noise ratio (SNR) of $5 \mathrm{~dB}$, there is almost no flash in the negative Doppler direction. This is because the front part of the round shape in the airfoil of the wing has a larger radar cross section (RCS) than the rear part of the pointed shape. Moreover, despite the change in $z$ values, the change in the spectrogram is insignificant in both pictures. Therefore, it is not possible to obtain the change in $z$ from the images. Fig. 8 illustrates the results of calculating the

Table 4. Parameters of the numerical simulation

\begin{tabular}{cc}
\hline Parameter & Value \\
\hline$L$ & $1 \mathrm{~m}$ \\
$f$ & $0.25 \mathrm{rps}$ \\
$f_{s}$ & $24 \mathrm{GHz}$ \\
$P$ & $600 \mathrm{sps}$ \\
$t_{P}$ & 36,000 \\
\hline
\end{tabular}

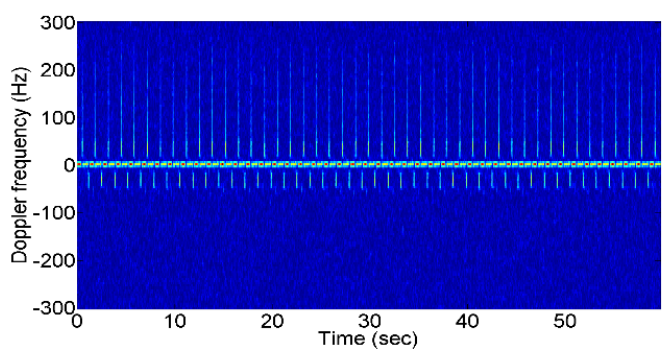

(a)

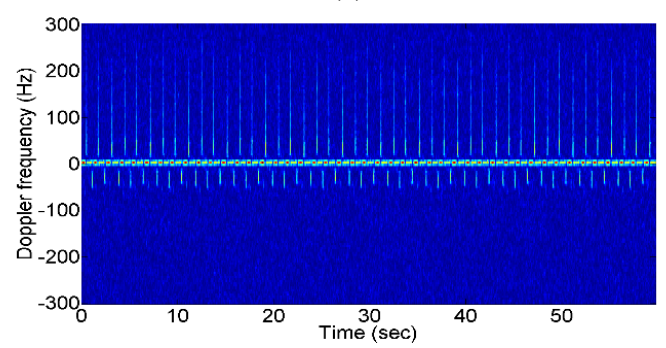

(b)

Fig. 7. Spectrogram obtained from the numerical data with $\mathrm{SNR}=$ $5 \mathrm{~dB}$ : (a) $z=0.1 \mathrm{rad} / \mathrm{sec}^{2}$ and (b) $z=0.3 \mathrm{rad} / \mathrm{sec}^{2}$.

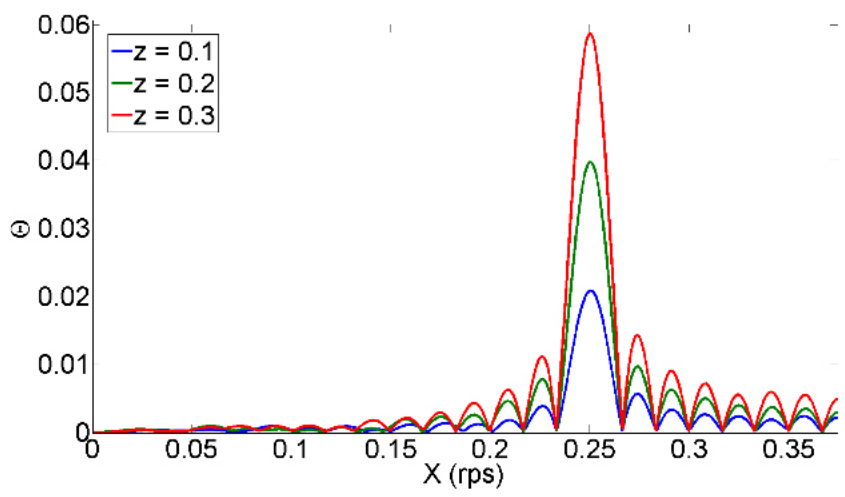

Fig. 8. Frequency spectrum for different $z\left(\mathrm{rad} / \mathrm{sec}^{2}\right)$ values with $\mathrm{SNR}=5 \mathrm{~dB}$.

Table 5. Estimated parameters of the numerical simulation with $X$ $=0.25 \mathrm{rps}$ and $\mathrm{SNR}=5 \mathrm{~dB}$

\begin{tabular}{ccc}
\hline $\begin{array}{c}\text { True } z \\
\left(\mathrm{rad} / \mathrm{sec}^{2}\right)\end{array}$ & $\begin{array}{c}\text { Estimated } z \\
\left(\mathrm{rad} / \mathrm{sec}^{2}\right)\end{array}$ & $\begin{array}{c}\text { Relative error for } z \\
(\%)\end{array}$ \\
\hline 0.1 & 0.1028 & 2.8 \\
0.2 & 0.1961 & 1.95 \\
0.3 & 0.2883 & 3.9 \\
\hline
\end{tabular}

frequency spectrum by applying the proposed method to the images in Fig. 7. Table 5 shows the $z$ extracted by the proposed method and their relative errors. From Table 5, the estimated $z$ shows an error below $4 \%$ compared to the actual $z$.

We used the additive Gaussian noise to represent a noisy signal. In this paper, SNR is defined based on the signal power excluding DC components. Fig. 9 shows the relative error of the proposed method calculated by 5,000 times Monte-Carlo simulation. The relative error is given by:

$$
\mathrm{RE}=\frac{|S-N|}{S} \times 100(\%)
$$

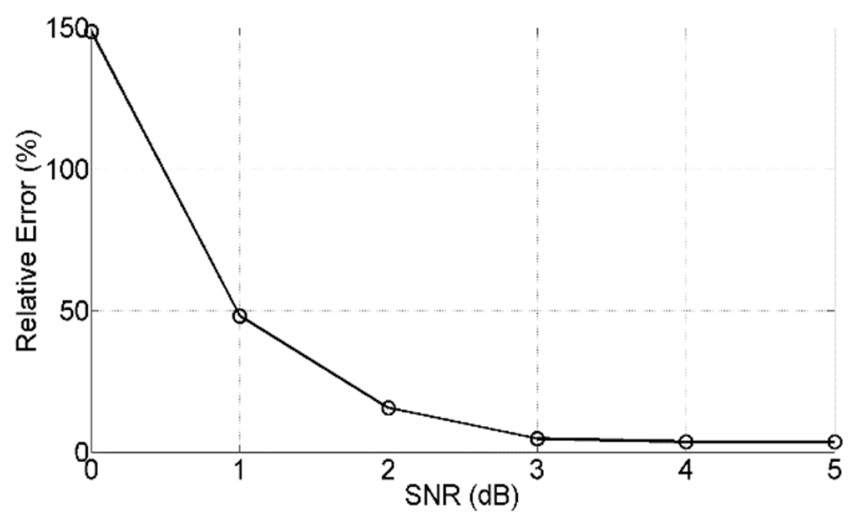

Fig. 9. Relative error of the proposed method calculated by 5,000 times Monte-Carlo simulation; $z=0.3\left(\mathrm{rad} / \mathrm{sec}^{2}\right)$. 
where $S$ is the signal power, and $N$ is the noise power. Fig. 10 shows the error of $z$ estimated by the proposed method in a frequency range from $1 \mathrm{GHz}$ to $24 \mathrm{GHz}$.

In Fig. 10, it is shown that the errors of $z$ estimated by the proposed method are almost the same, from $1 \mathrm{GHz}$ to $24 \mathrm{GHz}$. These results suggest that the proposed method has equal performance regardless of frequency.

\section{DISCUSSION AND CONCLUSION}

In this study, we proposed a method to detect the imbalance of the rotational speed and the rotational acceleration of wind turbines using the Doppler radar. The proposed method can accurately estimate the rotational speed of the wind turbine and the acceleration amplitude on the blade with an error within $2 \%$. Therefore, it is possible to detect the rotational speed imbalance of the wind turbine blade from the acceleration value of the blade. However, when $z=$ 0.01 , the error was found to increase significantly. This study primarily presented a method that can detect the imbalance of rotational speed using Doppler radar outside the wind turbine. While most sensors that determine the state of motion of a wind turbine acquire data from the blade rotation axis, Doppler radar can obtain data by directly observing the blade motion. Thus, the Doppler radar can provide a simpler way of obtaining data compared to the existing sensors. It is suggested that adding a Doppler radar to the existing condition monitoring system to observe the blades of a wind turbine is useful for diagnosing faults in the wind turbine.

Although the performance of the proposed method has been verified through simulation, there is a problem in practice that requires consideration. The power received by rainfall clutter is proportional to the square of the frequency. It is known that, in a high-frequency system such as a 24$\mathrm{GHz}$ Doppler radar, weather clutter-rainfall or fog-can cause serious performance degradation. This problem can be

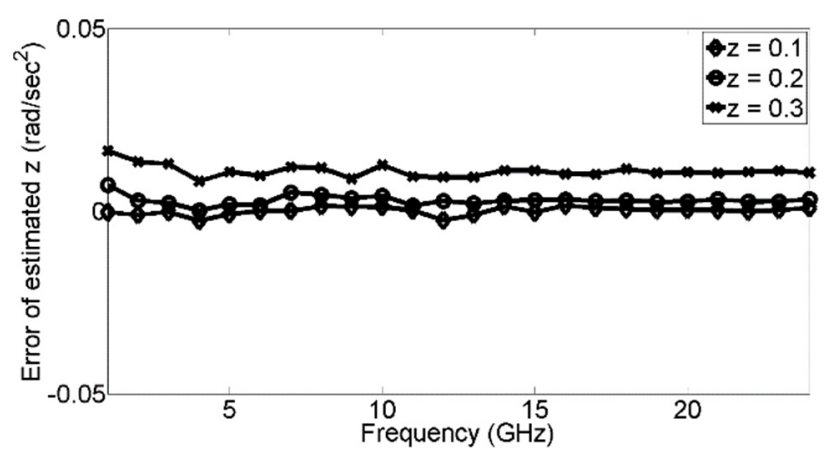

Fig. 10. Relative error of $z$ in a frequency range from $1 \mathrm{GHz}$ to $24 \mathrm{GHz}$ (noise free). avoided using a low-frequency system-L-band; however, low-frequency radar systems are more expensive than high frequency radar systems. Therefore, it is necessary to determine the optimum operating frequency by considering performance, economics, and portability.

When the rotor rotation speed is measured using a Doppler radar, the change in angular speed due to the imbalance of the rotor rotation speed is too small to determine the wind turbine failure. However, the change in the rotor rotation speed has a periodic pattern, which can be expressed as sinusoidal. Finally, the sinusoidal wave can be extracted using a Fourier transform. Using this principle, it is possible to estimate accurately the imbalance of the rotor rotation speed.

This research was supported by the Korea Electric Power Corporation (No. R19XO01-50). This work was also supported by the National Research Foundation of Korea (NRF) grant funded by the Korean government (Ministry of Science, ICT \& Future Planning) (No. NRF2018R1D1A1B07041496).

\section{REFERENCES}

[1] B. Lu, Y. Li, X. Wu, and Z. Yang, "A review of recent advances in wind turbine condition monitoring and fault diagnosis," in Proceedings of 2009 IEEE Power Electronics and Machines in Wind Applications, Lincoln, NE, 2009, pp. 1-7.

[2] S. A. Saleh and C. R. Moloney, "Development and testing of wavelet packet transform-based detector for ice accretion on wind turbines," in Proceedings of 2011 Digital Signal Processing and Signal Processing Education Meeting (DSP/SPE), Sedona, AZ, 2011, pp. 72-77.

[3] X. Gong and W. Qiao, "Imbalance fault detection of direct-drive wind turbines using turbine current signals," IEEE Transactions on Energy Conversion, vol. 27, no. 2, pp. 468-476, 2012.

[4] W. Yang, P. J. Tavner, and M. Wilkinson, "Wind turbine condition monitoring and fault diagnosis using both mechanical and electrical signatures," in Proceedings of 2008 IEEE/ASME International Conference on Advanced Intelligent Mechatronics, Xian, China, 2008, pp. 1296-1301.

[5] A. Naqvi, S. Yang, and H. Ling, "Investigation of Doppler features from wind turbine scattering," IEEE Antennas and Wireless Propagation Letters, vol. 9, pp. 485-488, 2010.

[6] F. Kong, Y. Zhang, R. Palmer, and Y. Bai, "Wind turbine radar signature characterization by laboratory measurements," in Proceedings of IEEE RadarCon (RADAR), Kansas City, MO, 2011, pp. 162-166. 
[7] F. Kong, Y. Zhang, and R. D. Palmer, "Wind turbine radar interference studies by polarimetric measurements of a scaled model," IEEE Transactions on Aerospace and Electronic Systems, vol. 49, no. 3, pp. 1589-1600, 2013.

[8] A. Naqvi and H. Ling, "Time-frequency and ISAR characteristics of wind turbines with higher order motions," Progress in Electromagnetics Research, vol. 143, pp. 331-347, 2013.

[9] J. Munoz-Ferreras, Z. Peng, Y. Tang, R. Gomez-Garcia, and C. Li, "Doppler-radar-based short-range acquisitions of time-frequency signatures from an industrial-type wind turbine," in Proceedings of IEEE Topical Conference on Wireless Sensors and Sensor Networks (WiSNet), Phoenix, AZ, 2017, pp. 5-7.

[10] T. Nikoubin, J. Munoz-Ferreras, R. Gomez-Garcia, D. Liang, and C. Li, "Structural health monitoring of wind turbines using a low-cost portable k-band radar: An abinitio field investigation," in Proceedings of 2015 IEEE Topical Conference on Wireless Sensors and Sensor Networks (WiSNet), San Diego, CA, 2015, pp. 69-71.

[11] J. Munoz-Ferreras, Z. Peng, Y. Tang, R. Gomez-Garcia, D. Liang, and C. Li, "Short-range Doppler-radar signatures from industrial wind turbines: theory, simulations, and measurements," IEEE Transactions on Instrumentation and Measurement, vol. 65, no. 9, 2108-2119, 2016.

[12] F. X. Ochieng, C. M. Hancock, G. W. Roberts, and J. Le Kernec, "A review of ground-based radar as a non con

\section{Young-Jae Choi}

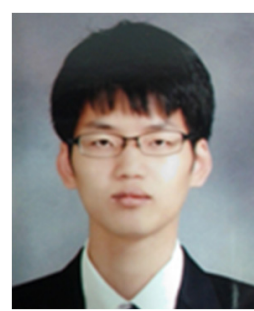

received B.S. and M.S. degrees in Electronics Engineering from Hannam University, Daejeon, Korea, in 2013 and 2018, respectively. He is working toward a Ph.D. degree in Electronics Engineering from Hannam University, Daejeon, Korea. His research interests include radar signal processing, RCS simulation and analysis. tact sensor for structural health monitoring of in-field wind turbines blades," Wind Energy, vol. 21, no. 12, pp. 1435-1449, 2018.

[13] P. Caselitz and J. Giebhardt, "Rotor condition monitoring for improved operational safety of offshore wind energy converters," Journal of Solar Energy Engineering, vol. 127, no. 2, pp. 253-261, 2005.

[14] P. Li, W. Hu, R. Hu, and Z. Chen, "Imbalance fault detection based on the integrated analysis strategy for variable-speed wind turbines," International Journal of Electrical Power \& Energy Systems, vol. 116, article no. 105570, 2020. https://doi.org/10.1016/j.ijepes.2019.105570.

[15] T. K. Moon and W. C. Stirling, Mathematical Methods and Algorithms for Signal Processing. Upper Saddle River, NJ: Prentice-Hall, 2000.

[16] N. A. Khan, M. N. Jafri, and S. A. Qazi, "Improved resolution short time Fourier transform," in Proceedings of the 7th International Conference on Emerging Technologies, Islamabad, Pakistan, 2011, pp. 1-3.

[17] V. C. Chen "Radar signatures of rotor blades," in Proceedings of SPIE 4391: Wavelet Applications VIII. Bellingham, WA: The International Society for Optical Engineering, 2001, pp. 63-70, 2001.

[18] C. Y. Kee and C. Wang, "Efficient GPU implementation of the high-frequency SBR-PO method," IEEE Antennas and Wireless Propagation Letters, vol. 12, pp. 941-944, 2013.

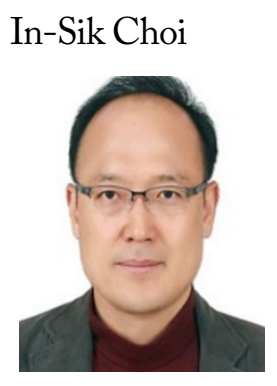

received a B.S. degree in Electrical and Electronics Engineering from Kyungpook National University, Daegu, Korea in 1998 and M.S. and Ph.D. degrees in Electrical and Electronics Engineering from Pohang University of Science and Technology (POSTECH), Pohang, Korea in 2000 and 2003, respectively. From 2004 to 2007, he worked at the Agency for Defense Development as a senior research engineer. He is now a Professor in the Department of Electrical and Electronics Engineering, Hannam University, Daejeon, Korea. His research interests include radar signal processing, RCS measurement and analysis, and EMI/EMC. 